\title{
Fatty Liver Index and Skeletal Muscle Density
}

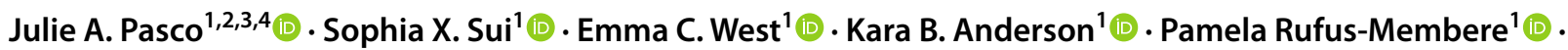

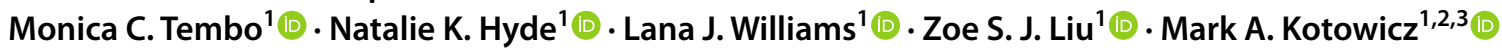

Received: 3 September 2021 / Accepted: 22 December 2021 / Published online: 13 January 2022

(c) The Author(s) 2022

\begin{abstract}
Accumulation of fat in the liver and skeletal muscle is associated with obesity and poor health outcomes. Liver steatosis is a characteristic of non-alcoholic fatty liver disease (NAFLD) and myosteatosis, of poor muscle quality in sarcopenia. In this study of 403 men (33-96 years), we investigated associations between the fatty liver index (FLI) and muscle density, as markers of fat accumulation in these organs. We also investigated associations between the FLI and parameters of sarcopenia, including DXA-derived appendicular lean mass (ALM) and handgrip strength by dynamometry. Muscle density was measured using pQCT at the radius and tibia. FLI was calculated from BMI, waist circumference, and levels of triglycerides and gamma-glutamyltransferase. There was a pattern of decreasing muscle density across increasing quartiles of FLI. After adjusting for age and lifestyle, mean radial muscle density in Q4 was 2.1\% lower than Q1 $(p<0.001)$ and mean tibial muscle density was 1.8\% lower in Q3 and 3.0\% lower in Q4, compared to Q1 ( $p=0.022$ and $<0.001$, respectively). After adjusting for age and sedentary lifestyle, participants in the highest FLI quartile were sixfold more likely to have sarcopenia. In conclusion, our results suggest that fat accumulation in the liver co-exists with fat infiltration into skeletal muscle.
\end{abstract}

Keywords NAFLD $\cdot$ Skeletal muscle fat infiltration $\cdot$ Muscle density $\cdot$ Myosteatosis $\cdot$ Hepatic steatosis $\cdot$ Sarcopenia

\section{Introduction}

Deposition of ectopic fat in the liver (liver steatosis) and infiltration of fat into skeletal muscle (myosteatosis) are both associated with impaired physiological function [1] and poor health outcomes [2]. Liver steatosis is a characteristic of non-alcoholic fatty liver disease (NAFLD) [2], and myosteatosis is a characteristic of poor muscle quality in sarcopenia [3]. The fatty liver index (FLI) was developed as a clinical risk assessment tool for identifying NAFLD. As intramuscular fat content increases, muscle density decreases, as the radiological density of fat is lower than muscle [4-6].
Julie A. Pasco

julie.pasco@deakin.edu.au

Sophia X. Sui

sophia.sui@deakin.edu.au

Emma C. West

westem@deakin.edu.au

Kara B. Anderson

kbanders@deakin.edu.au

Pamela Rufus-Membere pamela.r@deakin.edu.au

Monica C. Tembo

m.tembo@deakin.edu.au

Natalie K. Hyde

natalie.hyde@deakin.edu.au

Lana J. Williams

1.williams@deakin.edu.au
Zoe S. J. Liu

zoe.liu@deakin.edu.au

Mark A. Kotowicz

mark.kotowicz@deakin.edu.au

1 Deakin University, IMPACT - Institute for Mental and Physical Health and Clinical Translation, Geelong, VIC, Australia

2 Department of Medicine - Western Health, The University of Melbourne, St Albans, VIC, Australia

3 Barwon Health, Geelong, VIC, Australia

4 Department of Epidemiology and Preventive Medicine, Monash University, Melbourne, VIC, Australia 
Sequelae of fat infiltration into skeletal muscle include muscle atrophy, reduced muscle mass and muscle dysfunction $[3,5,7,8]$, and higher mortality [9]. This is due, at least in part, to the adipose tissue in muscle producing and releasing adipokines and inflammatory factors that act locally and systemically [10]. These can be released into the circulation, where they contribute to low grade inflammation. Locally, the accumulation of intramuscular lipid disrupts cellular energy homeostasis and leads to reduced protein synthesis, calcium imbalance and loss of contractile function, strength and exercise capacity [10].

Whilst there is evidence to support skeletal muscle-liver crosstalk [11] and data to suggest an association between skeletal muscle volume and NAFLD [12-17], data looking at the association between liver fat and skeletal muscle fat are limited $[18,19]$. If such an association exists, it might suggest that there are common mechanisms underpinning sarcopenia and NAFLD disease severity [20].

We hypothesised that there would be an inverse relationship between FLI and skeletal muscle density. The aim of this study was to investigate the association between liver fat and muscle fat, using FLI and skeletal muscle density as surrogate markers of fat accumulation in these organs. We also investigated associations between the FLI and parameters of sarcopenia.

\section{Methods}

\section{Participants}

This cross-sectional analysis utilises data from men enrolled in the Geelong Osteoporosis Study (GOS), a populationbased prospective cohort study of men and women, set in southeastern Australia. The GOS recruited 1540 men at random from electoral rolls between 2001 and 2006, with $67 \%$ response. The inclusion criterion was a listing on the electoral roll as a resident of the Barwon Statistical Division; residency in the region for less than 6 months and/or inability to provide informed consent necessitated exclusion. Most $(\sim 98 \%)$ of the participants were white. Details of recruitment and characteristics of the cohort have been published elsewhere [21]. Follow-up assessments occurred 5, 6, and 15 years later. Data for this analysis were collected at the most recent follow-up, 2016-2020. From a potential 625 men who participated in this phase, 403 (ages 33-96 years) provided a blood sample and underwent anthropometry and other clinical assessments including valid peripheral quantitative computed tomography (pQCT), and were thus included in this analysis.

The study was approved by the Human Research Ethics Committee at Barwon Health. All participants provided written informed consent.

\section{Muscle Density}

Muscle density and cross-sectional area (CSA) were measured using pQCT (XCT 2000, Stratec Medizintechnik, Pforzheim, Germany). Standard transverse scans were performed at $66 \%$ of radial $(n=349)$ and tibial $(n=347)$ length, and scans were analysed using BonAlyse software (BonAlyse Oy, Jyvaskyla, Finland). The following density thresholds identified bones, muscle, and fat tissue: fat $<15 \mathrm{mg} / \mathrm{mm}^{3}$, muscle $15-180 \mathrm{mg} / \mathrm{mm}^{3}$, and bone $>180 \mathrm{mg} / \mathrm{mm}^{3}$.

\section{Other Clinical Measures}

Body mass was measured to $\pm 0.1 \mathrm{~kg}$ using electronic scales and height was measured to $\pm 0.01 \mathrm{~m}$ using a wallmounted Harpenden stadiometer; body mass index (BMI) was calculated as body mass $/$ height ${ }^{2}\left(\mathrm{~kg} / \mathrm{m}^{2}\right)$. Waist circumference was measured in a horizontal plane with a narrow, non-elastic tape measure.

Handgrip strength (HGS) was measured using a handheld dynamometer (Vernier, LoggerPro3, USA). The maximum value of repeated measures for each hand was used in analyses and values transformed to Jamar equivalent values as previously described [22]. Gait speed was measured over a distance of $4 \mathrm{~m}$. The Timed Up-\&-Go (TUG) test over a distance of $3 \mathrm{~m}$ was used as a measure of balance and functional mobility [23]. Falls during the previous 12 months were documented by questionnaire and fallers were identified if one or more falls were reported.

Whole body dual energy x-ray absorptiometry (DXA; Prodigy Pro, Lunar, Madison, WI, USA) was used to determine whole body lean and fat mass. Lean mass of the arms and legs were summed to represent appendicular lean mass (ALM), which was expressed relative to height (ALM $/ \mathrm{h}^{2}$, $\mathrm{kg} / \mathrm{m}^{2}$ ) or BMI (ALM/BMI, $\mathrm{m}^{2}$ ). Body fat was expressed as a percentage of total body tissue (\% $\mathrm{BF})$. The android-togynoid ratio (AGR) was calculated as the ratio of android fat mass (the central region around the waist) and gynoid fat mass (mainly around the hips and thighs). All clinical measures were performed by trained personnel.

\section{Biomarkers}

Blood samples were collected following an overnight fast and sera were stored at $-80{ }^{\circ} \mathrm{C}$ until batch analysis. Serum triglycerides, gamma-glutamyltransferase (GGT), and plasma glucose were analysed using standard laboratory methods. Serum levels of interleukin (IL)- 6 and tumour necrosis factor (TNF)- $\alpha$ were assayed using a 
custom-designed Human High Sensitivity T Cell magnetic bead panel (MPHSTCMAG28SK05; Merk Millipore, Bayswater, VIC, AUS), in accordance with the manufacturer's instructions.

\section{Lifestyle}

Details of medication use and lifestyle were documented by self-report. Alcohol consumption was estimated using the Cancer Council Victoria food frequency questionnaire [24] and high intakes recognised if the average consumption exceeded $30 \mathrm{~g} /$ day [25]. Tobacco smoking referred to current use. Mobility was described as 'active' if vigorous or light exercise was performed regularly; otherwise individuals were classified as 'sedentary', as previously described [21]. Diabetes was identified by fasting plasma glucose $\geq 7.0 \mathrm{mmol} / \mathrm{L}$, use of an antihyperglycemic agent and/or self-report.

\section{Fatty Liver Index}

We used the Fatty Liver Index (FLI) as an indicator of liver fat accumulation [26]. The FLI was calculated from measures of BMI, waist circumference, triglycerides, and GGT to produce a score between 1 and 100 as follows: inflammatory markers (natural log transformed to normalise the data); models for muscle density were also adjusted for muscle CSA and ALM. Final models were tested for effect modification. The association between FLI (quartiles) and muscle density were repeated in two sensitivity analyses that excluded potential effects of diabetes and a high alcohol intake on this relationship. All analyses were performed using Minitab (v16, Minitab, State College, PA, USA).

\section{Results}

\section{Sample Characteristics}

The range of FLI values in each quartile was Q1 (lowest quartile, $n=101) 0.04-0.60, \mathrm{Q} 2(n=101) 0.61-1.75, \mathrm{Q} 3$ $(n=101)$ 1.76-4.97, and Q4 $(n=100)$ 5.35-84.0.

Participant characteristics are shown in Table 1. Indices of adiposity, including mean BMI, waist circumference, fat mass, \%BF, AGR, and median triglyceride and GGT levels increased across increasing FLI quartiles; no interquartile differences were detected in median age. The proportions of participants with diabetes increased across increasing FLI quartiles and this was also the pattern for sedentary

$\mathrm{FLI}=\left(e^{0.953 * \log e(\text { triglycerides })+0.139 * \mathrm{BMI}+0.718 * \log e(\mathrm{GGT})+0.053 * \text { waist circumference }-15.745}\right) /\left(1+e^{0.953 * \log e(\text { triglycerides })+0.139 * \mathrm{BMI}+0.718 * \log e(\mathrm{GGT})+0.053 * \text { waist circumference }-15.745}\right) * 100$

The distribution of FLI values were skewed, so the values were categorised into quartiles using the cut-points 0.597 , 1.759 , and 4.971 , which corresponded to $25 \%, 50 \%$, and $75 \%$ of the distribution; quartile (Q) 1 was designated low FLI. A FLI $<30$ (negative likelihood ratio $=0.2$ ) rules out and a $F L I \geq 60$ (positive likelihood ratio $=4.3$ ) rules in NAFLD.

\section{Statistical Methods}

Aggregate descriptive statistics were used to describe participant characteristics, and differences between FLI quartiles were identified using analysis of variance (ANOVA) where continuous data were normally distributed and Mann-Whitney $U$ analysis where continuous variables deviated from the normal distribution. The Chi-square test was used to identify differences between categorical data. Linear regression models (analysis of covariance, ANCOVA) were developed to determine how muscle density at the radial and tibial sites, ALM $/ \mathrm{h}^{2}$, ALM/BMI and HGS differed across quartiles of FLI. Binary logistic regression models were utilised to detect the likelihood of sarcopenia (based on low ALM/ BMI and low HGS) for each quartile of FLI. Potential covariates included age, high alcohol iontake, smoking, sedentary lifestyle, use of lipid-lowering medication, and serum lifestyles, slow TUG times, use of a lipid-lowering medication, and a high alcohol intake. No interquartile differences were observed for individuals with a fall in the previous 12 months.

\section{FLI and Components of Sarcopenia}

There was a pattern of increasing $\mathrm{ALM} / \mathrm{h}^{2}$, and decreasing ALM/BMI, across FLI quartiles (Table 2). The relationships between increasing FLI quartiles with increasing ALM/ $\mathrm{h}^{2}$ and decreasing ALM/BMI persisted after adjusting for age and sedentary lifestyle (Table 3). No interquartile differences in HGS were observed.

Eight participants (2.0\%) had both low HGS and low $\mathrm{ALM} / \mathrm{h}^{2}$, fulfilling criteria for sarcopenia (Table 2). The numbers of participants in each FLI quartile for sarcopenia using these criteria were too small for meaningful analyses. However, 40 participants (10.0\%) had both low HGS and low ALM/BMI. Using these criteria for sarcopenia revealed that participants in the highest FLI quartile (Q4) were sixfold more likely to have sarcopenia than those in the lowest quartile (Q1) $(p=0.004)$. No other interquartile differences were observed. With Q1 as reference, and models adjusted 
Table 1 Participant characteristics for the whole group and according to quartiles of fatty liver index (FLI)

\begin{tabular}{|c|c|c|c|c|c|c|}
\hline & \multirow{2}{*}{$\begin{array}{l}\text { All } \\
n=403\end{array}$} & \multicolumn{5}{|c|}{ Fatty liver index (FLI) } \\
\hline & & $\begin{array}{l}\text { Q1 } \\
n=101\end{array}$ & $\begin{array}{l}\text { Q2 } \\
n=101\end{array}$ & $\begin{array}{l}\text { Q3 } \\
n=101\end{array}$ & $\begin{array}{l}\mathrm{Q} 4 \\
n=100\end{array}$ & $p$ \\
\hline Age (year) & $64.7(54.0-73.5)$ & $62.6(49.3-74.6)$ & $63.2(55.2-72.7)$ & $66.1(55.1-72.8)$ & $67.0(56.3-73.6)$ & 0.287 \\
\hline BMI $\left(\mathrm{kg} / \mathrm{m}^{2}\right)$ & $27.8( \pm 4.0)$ & $23.6( \pm 1.9)$ & $26.6( \pm 1.9)$ & $28.4( \pm 1.8)$ & $32.5( \pm 3.7)$ & $<0.001$ \\
\hline Waist (cm) & $100( \pm 13)$ & $87( \pm 7)$ & $96( \pm 5)$ & $103( \pm 6)$ & $115( \pm 13)$ & $<0.001$ \\
\hline Triglycerides (mmol/L) & $1.2(0.9-1.8)$ & $0.9(0.7-1.1)$ & $1.1(0.9-1.5)$ & $1.5(1.0-2.0)$ & $2.0(1.5-2.6)$ & $<0.001$ \\
\hline GGT (U/L) & $24(17-35)$ & $16(13-23)$ & $22(17-28)$ & $27(20-39)$ & $35(25-63)$ & $<0.001$ \\
\hline IL-6 (pg/mL) & $1.40(0.73-2.54)$ & $1.58(0.73-2.91)$ & $1.27(0.68-2.41)$ & $1.39(0.92-2.48)$ & $1.47(0.69-2.31)$ & 0.514 \\
\hline TNF- $\alpha(\mathrm{pg} / \mathrm{mL})$ & $4.09(3.18-5.18$ & $3.75(2.83-5.00)$ & $4.13(3.38-5.09)$ & $4.10(3.13-5.31)$ & $4.22(3.05-5.36)$ & 0.608 \\
\hline Fat mass $(\mathrm{kg})$ & $25.4( \pm 9.4)$ & $15.3( \pm 4.9)$ & $23.3( \pm 4.9)$ & $27.0( \pm 5.1)$ & $36.0( \pm 8.0)$ & $<0.001$ \\
\hline$\% \mathrm{BF}(\%)$ & $30.1( \pm 7.5)$ & $21.8( \pm 6.0)$ & $29.2( \pm 5.1)$ & $32.3( \pm 4.6)$ & $37.1( \pm 4.5)$ & $<0.001$ \\
\hline AGR & $0.70( \pm 0.17)$ & $0.57( \pm 0.17)$ & $0.70( \pm 0.13)$ & $0.75( \pm 0.15)$ & $0.79( \pm 0.14)$ & $<0.001$ \\
\hline Diabetes* & $44(11.5 \%)$ & $5(5.3 \%)$ & $3(3.2 \%)$ & $19(20.0 \%)$ & $17(17.5 \%)$ & $<0.001$ \\
\hline Use of lipid-lowering medication & $113(28.0 \%)$ & $14(13.9 \%)$ & $28(27.7 \%)$ & $30(29.7 \%)$ & $41(41.0 \%)$ & $<0.001$ \\
\hline Smokers & $28(6.9 \%)$ & $9(8.9 \%)$ & $8(7.9 \%)$ & $9(8.9 \%)$ & $2(2.0 \%)$ & 0.162 \\
\hline Alcohol > 30 g/day & $81(20.1 \%)$ & $9(8.9 \%)$ & $23(22.8 \%)$ & $23(22.8 \%)$ & $26(26.0 \%)$ & 0.012 \\
\hline Sedentary behaviour & $96(23.8 \%)$ & $15(14.9 \%)$ & $17(16.8 \%)$ & $28(27.7 \%)$ & $36(36.0 \%)$ & 0.001 \\
\hline Gait speed $(\mathrm{m} / \mathrm{s})$ & $0.96( \pm 0.21)$ & $1.00( \pm 0.23)$ & $0.99( \pm 0.21)$ & $0.94( \pm 0.20)$ & $0.90( \pm 0.20)$ & 0.003 \\
\hline Gait speed $*<0.8 \mathrm{~m} / \mathrm{s}$ & $48(12.0 \%)$ & $11(10.9 \%)$ & $8(8.1 \%)$ & $13(13.0 \%)$ & $16(16.0 \%)$ & 0.367 \\
\hline TUG (s) & $8.4(7.5-9.8)$ & $8.0(7.2-9.1)$ & $8.1(7.3-9.3)$ & $8.4(7.8-10.0)$ & $9.2(8.0-10.8)$ & $<0.001$ \\
\hline TUG $>10 \mathrm{~s}$ & $62(15.4 \%)$ & $12(11.9 \%)$ & $7(6.9 \%)$ & $16(15.8 \%)$ & $27(27.0 \%)$ & 0.001 \\
\hline Faller & $54(13.4 \%)$ & $10(9.9 \%)$ & $11(10.9 \%)$ & $16(15.8 \%)$ & $17(17.0 \%)$ & 0.355 \\
\hline
\end{tabular}

Data are shown as mean ( \pm standard deviation), median (interquartile range) or $n(\%)$

$B M I$ body mass index; $G G T$ gamma-glutamyltransferase; $A G R$ android fat mass to gynoid fat mass ratio; $\% B F$ body fat percentage

*Missing data: diabetes $n=21$; gait speed $n=3$

Table 2 Skeletal muscle characteristics for the whole group and according to quartiles of fatty liver index (FLI)

\begin{tabular}{|c|c|c|c|c|c|c|}
\hline & \multirow{2}{*}{$\begin{array}{l}\text { All } \\
n=403\end{array}$} & \multicolumn{5}{|c|}{ Fatty liver index (FLI) } \\
\hline & & $\begin{array}{l}\text { Q1 } \\
n=101\end{array}$ & $\begin{array}{l}\mathrm{Q} 2 \\
n=101\end{array}$ & $\begin{array}{l}\mathrm{Q} 3 \\
n=101\end{array}$ & $\begin{array}{l}\mathrm{Q} 4 \\
n=100\end{array}$ & $p$ \\
\hline Radius: muscle density $\left(\mathrm{mg} / \mathrm{cm}^{3}\right)$ & $76.21( \pm 2.77)$ & $76.96( \pm 2.39)$ & $76.58( \pm 2.36)$ & $76.42( \pm 2.19)$ & $74.92( \pm 3.50)$ & $<0.001$ \\
\hline Tibia: muscle density $\left(\mathrm{mg} / \mathrm{cm}^{3}\right)$ & $71.51( \pm 4.59)$ & $73.05( \pm 4.13)$ & $71.85( \pm 4.33)$ & $71.06( \pm 4.37)$ & $69.85( \pm 5.02)$ & $<0.001$ \\
\hline Radius: muscle CSA $\left(\mathrm{cm}^{2}\right)$ & $41.53( \pm 6.45)$ & $39.13( \pm 6.40)$ & $40.71( \pm 5.98)$ & $41.40( \pm 5.48)$ & $44.79( \pm 6.58)$ & $<0.001$ \\
\hline Tibia: muscle CSA $\left(\mathrm{cm}^{2}\right)$ & $71.24( \pm 10.51)$ & $67.84( \pm 10.31)$ & $70.84( \pm 9.91)$ & $71.32( \pm 9.84)$ & $75.41( \pm 10.82)$ & $<0.001$ \\
\hline $\operatorname{ALM}(\mathrm{kg})$ & $26.0( \pm 3.8)$ & $24.5( \pm 3.5)$ & $26.0( \pm 3.8)$ & $25.8( \pm 3.2)$ & $27.5( \pm 4.0)$ & $<0.001$ \\
\hline $\operatorname{ALM} / \mathrm{h}^{2}\left(\mathrm{~kg} / \mathrm{m}^{2}\right)$ & $8.49( \pm 0.94)$ & $8.04( \pm 0.85)$ & $8.41( \pm 0.92)$ & $8.54( \pm 0.79)$ & $8.99( \pm 0.95)$ & $<0.001$ \\
\hline Low ALM/h ${ }^{2}$ & $16(4.0 \%)$ & $9(8.9 \%)$ & $3(2.0 \%)$ & $3(2.0 \%)$ & $2(2.0 \%)$ & - \\
\hline $\operatorname{ALM} / \mathrm{BMI}\left(\mathrm{m}^{2}\right)$ & $0.95( \pm 0.15)$ & $1.04( \pm 0.14)$ & $0.98( \pm 0.13)$ & $0.91( \pm 0.11)$ & $0.85( \pm 0.13)$ & $<0.001$ \\
\hline Low ALM/BMI & $82(20.4 \%)$ & $6(5.9 \%)$ & $10(9.9 \%)$ & $22(21.8 \%)$ & $44(44.0 \%)$ & $<0.001$ \\
\hline HGS $(\mathrm{kg})$ & $40.1( \pm 7.2)$ & $40.5( \pm 7.5)$ & $41.2( \pm 6.9)$ & $39.3( \pm 6.8)$ & $39.3( \pm 7.3)$ & 0.179 \\
\hline Low HGS* & $111(27.6 \%)$ & $27(27.0 \%)$ & $20(19.8 \%)$ & $31(30.7 \%)$ & $33(33.0 \%)$ & 0.169 \\
\hline Low HGS and low ALM $/ \mathrm{h}^{2 *}$ & $8(2.0 \%)$ & $5(5.0 \%)$ & $2(2.0 \%)$ & $0(0 \%)$ & $1(1.0 \%)$ & - \\
\hline Low HGS and low ALM/BMI* & $40(10.0 \%)$ & $4(4.0 \%)$ & $6(5.9 \%)$ & $9(8.9 \%)$ & $21(21.0 \%)$ & $<0.001$ \\
\hline
\end{tabular}

Low values: $\mathrm{HGS}<35.5 \mathrm{~kg} ; \mathrm{ALM} / \mathrm{h}^{2}\left(<6.94 \mathrm{~kg} / \mathrm{m}^{2}\right)$, ALM/BMI $\left(<0.827 \mathrm{~m}^{2}\right)$

CSA cross-sectional area; ALM appendicular lean mass; BMI body mass index; HGS handgrip strength

*Missing data: radius muscle density and CSA $n=54$; tibia muscle density and CSA $n=56$; HGS $n=1$ 
Table 3 Linear regression models showing the relationship between fatty liver index (FLI) quartiles (Q1 the lowest, and reference), appendicular lean mass, handgrip strength, and muscle density at the radial and tibial sites.

FLI Beta coefficient SE $p$

Radius: muscle density (mg/ Q1 Reference $\mathrm{cm}^{3}$ )

\begin{tabular}{lllll} 
& Q2 & -0.2589 & 0.3457 & 0.454 \\
& Q3 & -0.2349 & 0.3429 & 0.494 \\
& Q4 & -1.6623 & 0.3731 & 0.000 \\
$\begin{array}{l}\text { Tibia: muscle density }(\mathrm{mg} / \\
\left.\mathrm{cm}^{3}\right)\end{array}$ & Q1 & Reference & & \\
& & & & \\
& Q2 & -0.3969 & 0.5442 & 0.466 \\
& Q3 & -1.0329 & 0.5558 & 0.064 \\
& Q4 & -1.6463 & 0.6067 & 0.007 \\
ALM $/ \mathrm{h}^{2}\left(\mathrm{~kg} / \mathrm{m}^{2}\right)$ & Q1 & Reference & & \\
& Q2 & 0.4356 & 0.1106 & 0.000 \\
& Q3 & 0.6199 & 0.1111 & 0.000 \\
ALM/BMI $\left(\mathrm{m}^{2}\right)$ & Q4 & 1.1094 & 0.1125 & 0.000 \\
& Q1 & Reference & & \\
& Q2 & -0.05140 & 0.01530 & 0.000 \\
& Q3 & -0.11038 & 0.01538 & 0.000 \\
& Q4 & -0.16181 & 0.01557 & 0.000 \\
& Q1 & Reference & & \\
Handgrip strength $(\mathrm{kg})$ & Q2 & 1.4482 & 0.8397 & 0.085 \\
& Q3 & -0.0707 & 0.8436 & 0.933 \\
& Q4 & 0.1813 & 0.8538 & 0.832 \\
\hline & & & &
\end{tabular}

Muscle density models were adjusted for muscle CSA, ALM, age and sedentary lifestyle. Other models adjusted for age and sedentary lifestyle

$A L M$ appendicular lean mass; $B M I$ body mass index for age and lifestyle, the ORs (95\%CIs) for each quartile were: Q2 1.50 (0.37, 6.00); Q3 $1.91(0.51,7.09)$; and Q4 $6.01(1.77,20.4)$.

\section{FLI and Muscle Density}

There was a pattern of decreasing muscle density at the radial and tibial sites across increasing quartiles of FLI (Table 2). This pattern persisted after adjusting for age, sedentary lifestyle, ALM, and muscle CSA (Fig. 1). The associations were not explained by adjusting for use of a lipid-lowering medication, smoking or markers of inflammation. Compared with FLI QI, mean radial muscle density in Q4 was $2.1 \%$ lower $(p<0.001)$. Similarly, mean tibial muscle density was $2.2 \%$ lower in the highest compared to the lowest quartile $(p=0.006)$ and there was a trend for Q3 to be $0.5 \%$ lower than $\mathrm{Q} 1(p=0.06)$.

\section{Sensitivity Analyses}

In the first sensitivity analysis, 44 participants with diabetes were excluded. Amongst the remaining 359 participants, adjusted mean muscle density at the radius was lower for the highest FLI quintile compared with lowest $(p<0.001)$. Adjusted mean $(95 \% \mathrm{CI})$ for each FLI quartile, Q1 v Q2 v Q3 v Q4: $80.46(77.83,83.08)$ v $80.16(77.53,82.80) \mathrm{v}$ $80.46(77.77,83.15)$ v $78.81(76.07,81.55) \mathrm{mg} / \mathrm{cm}^{3}$. For the tibia, mean muscle density was lower for Q4 than Q1 $(p=0.027)$. The mean $(95 \% \mathrm{CI})$ values for each quartile of FLI: $75.01(71.54,78.48)$ v $74.84(71.33,78.35)$ v 74.30 $(70.71,77.89)$ v $73.63(69.99,77.26) \mathrm{mg} / \mathrm{cm}^{3}$.

In the second sensitivity analysis, 81 participants with high alcohol intake were excluded. Amongst the remaining 403 participants, adjusted mean muscle density at the
Fig. 1 Mean muscle density for each fatty liver index (FLI) quartile (Q); Q1 0.04-0.60, Q2 0.61-1.75, Q3 1.76-4.97, Q4 5.35-84.0. *Fatty Liver Index Q1 (reference) v Q4 $p \leq 0.007$; and $* * \mathrm{Q} 1$ (reference) $\mathrm{v} \mathrm{Q} 3, p=0.06$. The bars represent \pm standard error

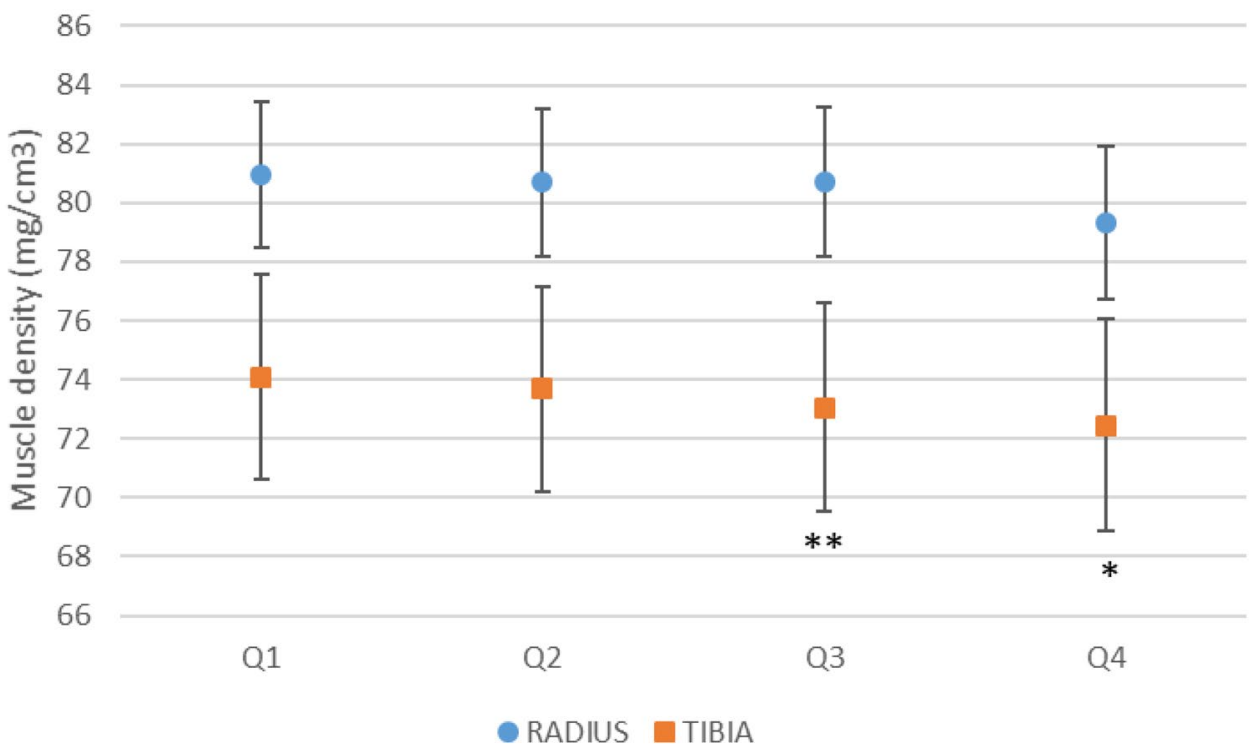


radius was lower for the highest FLI quintile compared with lowest $(p<0.001)$. Adjusted mean $(95 \% \mathrm{CI})$ for each FLI quartile, Q1 v Q2 v Q3 v Q4: $80.48(77.69,83.26)$ v $80.29(77.47,83.10)$ v $80.27(77.45,83.10)$ v $78.54(75.64$, $81.44) \mathrm{mg} / \mathrm{cm}^{3}$. For the tibia, in comparison to Q1, mean muscle density was lower for Q3 $(p=0.021)$ and lower for $\mathrm{Q} 4(p=0.001)$. The mean $(95 \% \mathrm{CI})$ values for each quartile of FLI: $74.94(71.02,78.85)$ v $74.52(70.53,78.51)$ v 73.53 $(69.54,77.52)$ v $72.65(68.58,76.72) \mathrm{mg} / \mathrm{cm}^{3}$.

\section{NAFLD and Muscle Density}

Amongst the 322 participants without high alcohol intake, four had FLI $\geq 60$ and met criteria for NAFLD, and five had FLI between 30 and 60. Median differences in radial muscle density for those with $(n=4)$ and without $(n=273)$ NAFLD were $75.25(70.81,78.28)$ v $76.61(74.99,78.16) \mathrm{mg} / \mathrm{cm}^{3}$, $p=0.477$; values of tibial muscle density for those with $(n=3)$ and without $(n=279)$ NAFLD were 73.83 (67.87, $73.83)$ v $72.49(69.30,74.72) \mathrm{mg} / \mathrm{cm}^{3}, p=0.677$. Numbers were too small for multivariable analyses.

\section{Discussion}

We report that men in the highest quartile of FLI had lower muscle density than those in the lowest quartile. Our results suggest that individuals likely to have more fat in the liver also have more fat in their muscle, at least at the radial and tibial regions that we measured. We also found that men in the highest quartile of FLI were more likely to have sarcopenia than those in the lowest quartile.

There is an emerging body of evidence to support links between skeletal muscle composition and chronic liver disease [20, 27]. Using different methodologies, several studies have reported low muscle volume in association with liver steatosis [12-17].

Both liver and muscle store carbohydrate as glycogen, and when storage capacities are overwhelmed, these organs convert excess carbohydrate into fat. Risk factors for fat accumulation in body organs include obesogenic environments, unhealthy lifestyles, and a genetic predisposition [28]. Whilst not limited to individuals with obesity, liver steatosis is more prevalent in those with metabolic aberrations, such as excessive visceral (central) fat accumulation and insulin resistance [2]. Combined low muscle volume and myosteatosis is more prevalent in NAFLD than in other chronic liver diseases [11]. NAFLD can progress to non-alcoholic steatohepatitis (NASH), which increases the risk for cirrhosis and hepatic carcinoma. Hepatic steatosis is interconnected with the metabolic syndrome and associated cardiometabolic diseases [2]; myosteatosis is also associated with chronic endocrine and non-endocrine disease [29], poor cognition [30], and poor mobility [31]. To date there has been little research to unravel potential pathophysiological links between hepatic steatosis and myosteatosis $[18,19]$, but underlying pathways are likely to involve lipotoxicity, insulin resistance, activated pathways for inflammation and oxidative stress, and mitochondrial dysfunction [28, 31-34].

In a study of 309 men and women (mean age $53 \pm 14$ years) from the Boramae NAFLD Registry in South Korea, individuals with sarcopenia, defined as low appendicular skeletal muscle index (measured by bioelectrical impedance analysis, BIA, and expressed as a percentage of body mass), were more likely to have NASH and significant fibrosis, independent of obesity, insulin resistance, and inflammation [20]. The authors concluded that sarcopenia was inversely associated with histological severity in NAFLD. A study using data from the National Health and Nutrition Examination Survey III (NHANES III) in the USA involving 2551 participants aged $60-75$ years identified NAFLD by ultrasound, and sarcopenia by low appendicular skeletal muscle index (by BIA; indexed to height or body mass) and function [35]. They reported that severe hepatic steatosis was inversely associated with sarcopenia using height-adjusted skeletal muscle index $\left(\mathrm{kg} / \mathrm{m}^{2}\right)$, but positively associated with sarcopenia using weight-adjusted skeletal muscle index $(\mathrm{kg} / \mathrm{kg})$.

A recent report from the Rotterdam Study described the inter-relationships between DXA-derived lean mass, body fat mass and distribution, and ultrasound-derived liver steatosis and NAFLD in 4609 elderly men and women from the Netherlands [36]. They reported that body fat mass, particularly android fat mass, was a better predictor than low lean mass for NAFLD. A negative association between ALM $/ \mathrm{h}^{2}$ and NAFLD was independent of metabolic confounders and fat distribution in normal-weight women, but not men. No association was observed between sarcopenia and NAFLD. In our cross-sectional analysis of data for men, we report that indices of adiposity, central fat accumulation and ALM $/ \mathrm{h}^{2}$ increased, and ALM/BMI decreased, across increasing quartiles of FLI. No association was detected between quartiles of FLI and handgrip strength or gait speed.

The absence of an internationally accepted operational definition for sarcopenia has led to inconsistencies in the way sarcopenia is identified [37]. In particular, diagnostic methods for low muscle quantity include total lean mass or ALM adjusted for height $\left(\mathrm{kg} / \mathrm{m}^{2}\right)$, BMI $\left(\mathrm{m}^{2}\right)$, or body mass (\%), and not all definitions include parameters of muscle function. Where muscle dysfunction attributed to low muscle volume occurs in the face of obesity, the combination is referred to as sarcopenic obesity [38, 39]; age-related muscle deterioration accompanied by increases in body fat mass has been identified [40]. Peng et al. [35, 41] noted that BMI confounds the relationship between sarcopenia and NAFLD 
in studies using different definitions for sarcopenia. We also know that BMI is limited as an estimate of body fatness, and it varies with age, sex, and ethnicity $[42,43]$.

In this study, we evaluated skeletal muscle density using pQCT. This imaging technology evolved from the established computed tomography (CT) to quantify bone parameters at peripheral sites and, more recently, for quantifying muscle mass and fat distribution. In muscles, both modalities use $\mathrm{x}$-ray beam attenuation to distinguish fat from fat-free mass. CT-derived muscle density at the mid-thigh has been negatively correlated with lipid content from percutaneous biopsy specimens [3]. As with CT, pQCT determines the CSA of soft tissue and estimates muscle density. The main advantage of using pQCT instead of CT is the extremely low effective radiation dose and shorter scan time [44], making it suitable for epidemiological studies.

We acknowledge several strengths and weaknesses in our study. Particular strengths are that participants were representative of the general adult population and not selected on the basis of disease. We did not have a direct measure of liver fat, but used the FLI as a surrogate. In a study showing that the FLI discriminated between patients with and without liver steatosis, a poor relationship was reported between FLI and liver fat measured by proton magnetic resonance spectroscopy [45]. On the other hand, a recent communitybased study indicated a correlation $(r=0.56-0.59)$ between liver fat estimated by ultrasound and the FLI [46]. We used DXA-derived ALM as a surrogate for skeletal muscle mass which has been shown to indicate a correlation $(r=0.91$ for the leg) with skeletal muscle mass quantified by magnetic resonance imaging [47]. The use of the FLI to stratify participants is a potential limitation in analyses involving indices of adiposity, for example ALM/BMI, \%BF, waist circumference, as they are part of the FLI. The FLI stratification may have also limited our ability to identify associations between inflammatory markers, hepatic steatosis, and myosteatosis. Furthermore, our results are likely to depend on the definitions and cut-points selected a priori for identifying low values for muscle parameters and sarcopenia for the secondary analyses regarding associations between FLI and muscle mass, strength, and performance. Although we have accounted for indices of adiposity in the FLI, there is likely to be residual confounding in our statistical models. Further, we used cross-sectional data, which cannot infer causality. Our data were pertinent for men residing in southeastern Australia, and may not be generalisable to other populations of men, nor to women.

Given these constraints, we conclude that fat accumulation in the liver co-exists with fat infiltration into skeletal muscle. The presence of abnormal liver function tests or NAFLD diagnosis should prompt assessment for sarcopenia and frailty (using muscle performance tests) as a clinical consequence of myosteatosis. On the other hand, identification of poor muscle function associated with low muscle density may indicate the presence of unrecognised hepatosteatosis. Storage of ectopic fat in liver and skeletal muscle, leading to fatty liver disease and compromised skeletal muscle, places individuals in a web of metabolic disturbances in combination with muscle deficits that are likely to increase the risk for poor physical function.

Acknowledgements The authors would like to thank Professor Graham Giles of the Cancer Epidemiology Centre of The Cancer Council Victoria, for permission to use the Dietary Questionnaire for Epidemiological Studies (v2), Melbourne: The Cancer Council Victoria 1996. Study data were collected and managed using REDCap electronic data capture tools hosted at the University Hospital Geelong (Barwon Health).

Author Contributions JAP and MAK designed the study. JAP was responsible for statistical analysis of the data and prepared the first draft of the paper; she is guarantor. JAP, LJW, and MAK were responsible for the funding acquisition. SXS, KBA, PR-M, MCT, and ZL contributed to the data acquisition. All authors agreed to the interpretation of the data, revised the paper critically for intellectual content, and approved the final version. All authors agree to be accountable for the work and to ensure that any questions relating to the accuracy and integrity of the paper are investigated and properly resolved.

Funding Open Access funding enabled and organised by CAUL and its Member Institutions. The Geelong Osteoporosis Study was funded by the National Health and Medical Research Council (NHMRC) Australia [Grant Nos. 299831, 628582]. SXS was supported by an Execute Dean's Postdoctoral Research Fellowship (Deakin University); ECW, KBA, PR-M and MCT by Australian Government Research Training Program Scholarships; NKH by a Dean's Research Postdoctoral Fellowship (Deakin University); and LJW by a NHMRC Career Development Fellowship (1064272) and a NHMRC Investigator grant (1174060).

Data Availability Data will be made available upon reasonable request.

Code Availability Not applicable.

\section{Declarations}

Conflict of interest The authors declare no conflict of interest.

Consent to Participate Informed consent was obtained from all participants involved in the study.

Consent for Publication Not applicable.

Ethical Approval The study was conducted according to the guidelines of the Declaration of Helsinki and approved by the Human Research Ethics Committee at Barwon Health.

Research Involving Humans and/or Animals Participants Not applicable.

Open Access This article is licensed under a Creative Commons Attribution 4.0 International License, which permits use, sharing, adaptation, distribution and reproduction in any medium or format, as long as you give appropriate credit to the original author(s) and the source, provide a link to the Creative Commons licence, and indicate if changes 
were made. The images or other third party material in this article are included in the article's Creative Commons licence, unless indicated otherwise in a credit line to the material. If material is not included in the article's Creative Commons licence and your intended use is not permitted by statutory regulation or exceeds the permitted use, you will need to obtain permission directly from the copyright holder. To view a copy of this licence, visit http://creativecommons.org/licenses/by/4.0/.

\section{References}

1. Carobbio S, Rodriguez-Cuenca S, Vidal-Puig A (2011) Origins of metabolic complications in obesity: ectopic fat accumulation. The importance of the qualitative aspect of lipotoxicity. Curr Opin Clin Nutr Metab Care 14(6):520-526

2. Arsenault BJ, Beaumont EP, Despres JP, Larose E (2012) Mapping body fat distribution: a key step towards the identification of the vulnerable patient? Ann Med 44(8):758-772

3. Goodpaster BH, Carlson CL, Visser M, Kelley DE, Scherzinger A, Harris TB, Stamm E, Newman AB (2001) Attenuation of skeletal muscle and strength in the elderly: the Health ABC Study. J Appl Physiol 90(6):2157-2165

4. Larson-Meyer DE, Smith SR, Heilbronn LK, Kelley DE, Ravussin E, Newcomer BR, Look AARG (2006) Muscle-associated triglyceride measured by computed tomography and magnetic resonance spectroscopy. Obesity (Silver Spring, MD) 14(1):73-87

5. Visser M, Kritchevsky SB, Goodpaster BH, Newman AB, Nevitt M, Stamm E, Harris TB (2002) Leg muscle mass and composition in relation to lower extremity performance in men and women aged 70 to 79: the Health, Aging and Body Composition study. J Am Geriatr Soc 50(5):897-904

6. Goodpaster BH, Kelley DE, Thaete FL, He J, Ross R (2000) Skeletal muscle attenuation determined by computed tomography is associated with skeletal muscle lipid content. J Appl Physiol 89(1):104-110

7. Ilich JZ, Kelly OJ, Inglis JE, Panton LB, Duque G, Ormsbee MJ (2014) Interrelationship among muscle, fat, and bone: connecting the dots on cellular, hormonal, and whole body levels. Ageing Res Rev 15:51-60

8. Visser M, Goodpaster BH, Kritchevsky SB, Newman AB, Nevitt M, Rubin SM, Simonsick EM, Harris TB (2005) Muscle mass, muscle strength, and muscle fat infiltration as predictors of incident mobility limitations in well-functioning older persons. $\mathrm{J}$ Gerontol A 60(3):324-333

9. Miljkovic I, Kuipers AL, Cauley JA, Prasad T, Lee CG, Ensrud KE, Cawthon PM et al (2015) Greater skeletal muscle fat infiltration is associated with higher all-cause and cardiovascular mortality in older men. J Gerontol A 70(9):1133-1140

10. Al Saedi A, Goodman CA, Myers D, Hayes A, Duque G (2019) Osteosarcopenia as a lipotoxic disease. In: Duque G (ed) Osteosarcopenia: bone, muscle and fat interactions. Springer, Cham, pp 123-143. ISBN 978-3-030-25889-4

11. Chakravarthy MV, Siddiqui MS, Forsgren MF, Sanyal AJ (2020) Harnessing muscle-liver crosstalk to treat nonalcoholic steatohepatitis. Front Endocrinol (Lausanne) 11:592373

12. Hsing JC, Nguyen MH, Yang B, Min Y, Han SS, Pung E, Winter SJ et al (2019) Associations between body fat, muscle mass, and nonalcoholic fatty liver disease: a population-based study. Hepatol Commun 3(8):1061-1072

13. Kim G, Lee SE, Lee YB, Jun JE, Ahn J, Bae JC, Jin SM et al (2018) Relationship between relative skeletal muscle mass and nonalcoholic fatty liver disease: a 7-year longitudinal study. Hepatology 68(5):1755-1768

14. Kim HY, Kim CW, Park CH, Choi JY, Han K, Merchant AT, Park YM (2016) Low skeletal muscle mass is associated with non-alcoholic fatty liver disease in Korean adults: the Fifth Korea National Health and Nutrition Examination Survey. Hepatobiliary Pancreat Dis Int 15(1):39-47

15. Lee YH, Jung KS, Kim SU, Yoon HJ, Yun YJ, Lee BW, Kang ES et al (2015) Sarcopaenia is associated with NAFLD independently of obesity and insulin resistance: nationwide surveys (KNHANES 2008-2011). J Hepatol 63(2):486-493

16. Hong HC, Hwang SY, Choi HY, Yoo HJ, Seo JA, Kim SG, Kim NH et al (2014) Relationship between sarcopenia and nonalcoholic fatty liver disease: the Korean Sarcopenic Obesity Study. Hepatology 59(5): 1772-1778

17. Moon JS, Yoon JS, Won KC, Lee HW (2013) The role of skeletal muscle in development of nonalcoholic fatty liver disease. Diabetes Metab J 37(4):278-285

18. Nachit M, Leclercq IA (2019) Emerging awareness on the importance of skeletal muscle in liver diseases: time to dig deeper into mechanisms! Clin Sci (Lond) 133(3):465-481

19. Hsu CS, Kao JH (2018) Sarcopenia and chronic liver diseases. Expert Rev Gastroenterol Hepatol 12(12):1229-1244

20. Koo BK, Kim D, Joo SK, Kim JH, Chang MS, Kim BG, Lee KL, Kim W (2017) Sarcopenia is an independent risk factor for non-alcoholic steatohepatitis and significant fibrosis. J Hepatol 66(1):123-131

21. Pasco JA, Nicholson GC, Kotowicz MA (2012) Cohort profile: Geelong Osteoporosis Study. Int J Epidemiol 41(6):1565-1575

22. Sui SX, Holloway-Kew KL, Hyde NK, Williams LJ, Tembo MC, Leach S, Pasco JA (2020) Definition-specific prevalence estimates for sarcopenia in an Australian population: the Geelong Osteoporosis Study. JCSM Clin Rep 5:89-98

23. Podsiadlo D, Richardson S (1991) The timed "Up \& Go": a test of basic functional mobility for frail elderly persons. J Am Geriatr Soc 39(2):142-148

24. Giles GG, Ireland PD (1996) Dietary questionnaire for epidemiological studies (version 2). The Cancer Council, Melbourne

25. Kanis JA, Johnell O, Oden A, Johansson H, McCloskey E (2008) FRAX and the assessment of fracture probability in men and women from the UK. Osteoporos Int 19(4):385-397

26. Bedogni G, Bellentani S, Miglioli L, Masutti F, Passalacqua M, Castiglione A, Tiribelli C (2006) The Fatty Liver Index: a simple and accurate predictor of hepatic steatosis in the general population. BMC Gastroenterol 6:33

27. Eslamparast T, Montano-Loza AJ, Raman M, Tandon P (2018) Sarcopenic obesity in cirrhosis-the confluence of 2 prognostic titans. Liver Int 38(10):1706-1717

28. Soto-Angona O, Anmella G, Valdes-Florido MJ, De Uribe-Viloria N, Carvalho AF, Penninx B, Berk M (2020) Non-alcoholic fatty liver disease (NAFLD) as a neglected metabolic companion of psychiatric disorders: common pathways and future approaches. BMC Med 18(1):261

29. Borba VZC, Costa TL, Moreira CA, Boguszewski CL (2019) Mechanisms of endocrine disease: sarcopenia in endocrine and non-endocrine disorders. Eur J Endocrinol 180(5):R185-R199

30. Sui SX, Williams LJ, Holloway-Kew KL, Hyde NK, Anderson KB, Tembo MC, Addinsall AB, Leach S, Pasco JA (2021) Skeletal muscle density and cognitive function: a cross-sectional study in men. Calcif Tissue Int 108(2):165-175

31. Morley JE, Anker SD, von Haehling S (2014) Prevalence, incidence, and clinical impact of sarcopenia: facts, numbers, and epidemiology-update 2014. J Cachexia Sarcopenia Muscle 5(4):253-259

32. Srikanthan P, Karlamangla AS (2011) Relative muscle mass is inversely associated with insulin resistance and prediabetes. 
Findings from the third National Health and Nutrition Examination Survey. J Clin Endocrinol Metab 96(9):2898-2903

33. Pasco JA (2019) Age-related changes in muscle and bone. In: Duque G (ed) Osteosarcopenia: bone, muscle and fat interactions. Springer, Cham. ISBN 978-3-030-25889-4

34. Schaap LA, Pluijm SM, Deeg DJ, Visser M (2006) Inflammatory markers and loss of muscle mass (sarcopenia) and strength. Am J Med 119(6):526 e529-517

35. Peng TC, Wu LW, Chen WL, Liaw FY, Chang YW, Kao TW (2019) Nonalcoholic fatty liver disease and sarcopenia in a Western population (NHANES III): the importance of sarcopenia definition. Clin Nutr 38(1):422-428

36. Alferink LJM, Trajanoska K, Erler NS, Schoufour JD, de Knegt RJ, Ikram MA, Janssen HLA et al (2019) Nonalcoholic fatty liver disease in the Rotterdam study: about muscle mass, sarcopenia, fat mass, and fat distribution. J Bone Miner Res 34(7):1254-1263

37. Zanker J, Scott D, Reijnierse EM, Brennan-Olsen SL, Daly RM, Girgis CM, Grossmann M et al (2019) Establishing an operational definition of sarcopenia in Australia and New Zealand: Delphi method based consensus statement. J Nutr Health Aging 23(1):105-110

38. Stenholm S, Harris TB, Rantanen T, Visser M, Kritchevsky SB, Ferrucci L (2008) Sarcopenic obesity: definition, cause and consequences. Curr Opin Clin Nutr Metab Care 11(6):693-700

39. Baumgartner RN (2000) Body composition in healthy aging. Ann N Y Acad Sci 904:437-448

40. Pasco JA, Gould H, Brennan SL, Nicholson GC, Kotowicz MA (2014) Musculoskeletal deterioration in men accompanies increases in body fat. Obesity (Silver Spring, MD) 22(3):863-867

41. Peng TC (2018) Role of sarcopenia in nonalcoholic fatty liver disease: definition is crucially important. Hepatology 68(2):788-789
42. Pasco JA, Nicholson GC, Brennan SL, Kotowicz MA (2012) Prevalence of obesity and the relationship between the body mass index and body fat: cross-sectional, population-based data. PLoS ONE 7(1):e29580

43. Deurenberg P, Deurenberg-Yap M, Guricci S (2002) Asians are different from Caucasians and from each other in their body mass index/body fat per cent relationship. Obes Rev 3(3):141-146

44. Erlandson MC, Lorbergs AL, Mathur S, Cheung AM (2016) Muscle analysis using pQCT, DXA and MRI. Eur J Radiol 85(8):1505-1511

45. Cuthbertson DJ, Weickert MO, Lythgoe D, Sprung VS, Dobson R, Shoajee-Moradie F, Umpleby M et al (2014) External validation of the fatty liver index and lipid accumulation product indices, using $1 \mathrm{H}$-magnetic resonance spectroscopy, to identify hepatic steatosis in healthy controls and obese, insulin-resistant individuals. Eur J Endocrinol 171(5):561-569

46. Chen LW, Huang PR, Chien CH, Lin CL, Chien RN (2020) A community-based study on the application of fatty liver index in screening subjects with nonalcoholic fatty liver disease. J Formos Med Assoc 119(1 Pt 1):173-181

47. Chen Z, Wang Z, Lohman T, Heymsfield SB, Outwater E, Nicholas JS, Bassford T et al (2007) Dual-energy X-ray absorptiometry is a valid tool for assessing skeletal muscle mass in older women. J Nutr 137(12):2775-2780

Publisher's Note Springer Nature remains neutral with regard to jurisdictional claims in published maps and institutional affiliations. 